THE AstrophysiCAL Journal, 567:1192-1201, 2002 March 10

(C) 2002. The American Astronomical Society. All rights reserved. Printed in U.S.A.

\title{
INCLUSION OF TURBULENCE IN SOLAR MODELING
}

\author{
L. H. Li, F. J. Robinson, P. Demarque, And S. Sofia \\ Department of Astronomy, Yale University, P.O. Box 208101, New Haven, CT 06520-8101 \\ AND \\ D. B. GUENTHER \\ Department of Astronomy and Physics, Saint Mary's University, Halifax, NS B3H 3C3, Canada \\ Received 2001 August 6; accepted 2001 October 29
}

\begin{abstract}
The general consensus is that in order to reproduce the observed solar $p$-mode oscillation frequencies, turbulence should be included in solar models. However, until now there has not been any well-tested efficient method to incorporate turbulence into solar modeling. We present here two methods to include turbulence in solar modeling within the framework of the mixing length theory, using the turbulent velocity obtained from numerical simulations of the highly superadiabatic layer (SAL) of the Sun at three stages of its evolution. The first approach is to include the turbulent pressure alone, and the second is to include both the turbulent pressure and the turbulent kinetic energy. The latter is achieved by introducing two variables: the turbulent kinetic energy per unit mass and the effective ratio of specific heats owing to the turbulent perturbation. These are treated as additions to the standard thermodynamic coordinates (e.g., pressure and temperature). We investigate the effects of both treatments of turbulence on the structure variables, the adiabatic sound speed, the structure of the highly superadiabatic layer, and the $p$-mode frequencies. We find that the second method reproduces the SAL structure obtained in three-dimensional simulations and produces a $p$-mode frequency correction an order of magnitude better than the first method.
\end{abstract}

Subject headings: Sun: evolution - Sun: helioseismology - Sun: interior - turbulence

\section{INTRODUCTION}

The effects of turbulence on the structure of a solar model depends not only on how it is modeled but also on how it is incorporated into the solar model. The change in the $p$-mode oscillation frequencies caused by turbulence can be used as a measure of the effects of turbulence on the structure of solar models, since helioseismology provides an opportunity to probe directly and sensitively solar structure. In the standard solar models (SSMs), the turbulent convection is modeled in terms of the local mixing-length theory (MLT), but the turbulent pressure, turbulent kinetic energy, and turbulent entropy are ignored. The fact that the computed frequencies of $p$-modes from SSMs are higher than the observed values shows that SSMs need to be refined. The frequency dependence of the discrepancy reveals that there is a problem with the model and that the problem lies very near the surface. Balmforth (1992a, 1992b, 1992c) uses the nonlocal MLT to model the turbulent convection and includes the turbulent pressure, but ignores the turbulent kinetic energy and turbulent entropy in modeling the Sun. The computed frequencies of $p$-modes from such a model are even higher than those computed from the SSMs (Balmforth 1992a). Canuto $(1990,1996)$ has proposed a semianalytical model of the turbulent convection. The main idea is to include a full turbulent spectrum in the model of convection. Applications to stellar models have been discussed by Canuto \& Mazzitelli $(1991,1992)$ and Canuto, Goldman, \& Mazzitelli (1996). The parameters of the Canuto-Mazzitelli (C\&M) approach are derived from the laboratory experiments of the incompressible convection and extrapolated to the stellar conditions. Using this approach, the superadiabatic peak is much higher than that of the SSMs, while the computed frequencies of solar $p$-modes are closer to the observed values than those from the SSMs.
Turbulence is a highly nonlinear phenomenon. Numerical experiments are a direct, effective way to investigate turbulence, in addition to the laboratory experiments (Niemela et al. 2000). Chan \& Sofia (1989) performed a three-dimensional numerical simulation of the deep compressible convection, where the superadiabatic excess in the temperature gradient is very small. They showed that in the deep regions, the mixing-length approximation is valid. They derived expressions for key physical parameters such as the convective energy flux. These, in principle, can be conveniently applied to stellar models. The Chan-Sofia formula for the convective flux was incorporated into the Yale stellar evolution code by Lydon (1993) and Lydon, Fox, \& Sofia (1992). The diffusion approximation was used to treat radiative transfer, and the radiative atmosphere was treated by Lydon et al. (1992) as in the standard MLT Yale models (e.g., Guenther et al. 1992). They found that the peak of the superadiabatic layer (SAL) is not as high as in the C\&M model and is located at a greater depth below the surface. However, their models yielded a larger discrepancy between the observed solar $p$-mode frequencies and those computed from SSMs. Part of the discrepancy could be attributed to the fact that these models did not match the solar radius very precisely. However, this is expected since the simulation, which is valid for the deeper adiabatic regions, is extrapolated into the SAL, where the temperature gradient greatly exceeds the adiabatic gradient, and some inaccuracy is inevitable.

In order to overcome these difficulties, Kim (1993) and Kim et al. $(1995,1996)$ conducted a three-dimensional numerical simulation whose domain includes shallower layers. This simulation treats the coupling of radiation and convection and includes realistic equation of state and radiative opacities, taken from the Yale stellar evolution code. Kim et al.'s $(1995,1996)$ simulation treats radiative transfer 
in the diffusion approximation, which is valid only in an optically thick medium, and consequently cannot be used in the solar atmosphere. Thus, the top boundary of the simulation was set below the SAL peak. Kim et al.'s (1996) models were parameterized as a varying mixing length with depth by Demarque, Guenther, \& Kim (1997), in precisely calibrated solar models. The $p$-mode frequencies of the models were found to agree more closely with the observed solar $p$-mode frequencies than the SSM frequencies, but these models exhibit, like the C\&M models, a higher SAL peak than the SSMs.

More recently, Kim \& Chan (1998) have completed a three-dimensional radiative hydrodynamic simulation of the complete extent of the SAL including the solar atmosphere (about 2 pressure scale heights above and 2.5 pressure scale heights below the SAL). The numerical approach was described by Kim \& Chan (1997). The simulation of Kim \& Chan is fully compressible and uses the realistic equation of state and opacities. The radiation has been treated by utilizing the three-dimensional Eddington approximation, which is valid in both the optically thin regions near the surface and the optically thick regions in the interior. Demarque et al. (1999) mimicked this simulation in the calibrated solar models by increasing the opacity coefficient $\kappa$ near the surface. Such models are called perturbed- $\kappa$ models. The discrepancy between calculated and observed $p$-mode frequencies decreases when compared to SSMs. A deeper layer (about 5 pressure scale heights above and 6 pressure scale heights below the SAL) was simulated by Stein \& Nordlund (1998) using different numerical methods for the convective and radiative components. Instead of parameterizing the simulation, Rosenthal et al. (1999) matched the simulation to an envelope that was constructed using an MLT envelope code. The computed frequencies are in better agreement with the observed solar $p$-mode frequencies when compared to the SSMs. Abbett et al. (1997) have also discussed the same transition layer.

One way to include turbulence in stellar models is to use the numerical simulations to directly calculate the convective temperature gradient and its derivatives needed in stellar model calculations. However, because turbulence is chaotic, nonlocal, and three-dimensional, and because it involves nonlinear interactions over many disparate length scales, the simulation for the whole convective zone is too computationally expensive for stellar model calculations. Besides, the accuracy of the simulation, if performed, is too low to match the required accuracy for the stellar model calculations. Fortunately, the existing simulations show that the MLT prediction deviates from simulations only in the SAL part of the convection zone. Even so, it is still impractical to calculate the convective temperature gradient and its derivatives, from numerical simulation of the SAL in the stellar evolution model calculations. Robinson et al. (2001) have performed some hydrodynamical simulations (the viscosity parameter $c_{\mu}=0.2 \sqrt{2}$; see below) of the SAL at three stages in the solar evolution: the zero-age main sequence (ZAMS), the present Sun, and the subgiant. The results show that the turbulent velocities in the SAL, as functions of gas pressure, change little for all these stages. Therefore, it is feasible to compute the effects of turbulence on the convective gradients. Overshooting was observed in both the solar subatmosphere and three-dimensional numerical simulations, and the $\kappa$-models (Demarque et al.
1999) demonstrate that overshooting is likely to be one of the keys to match the real Sun. It is a challenge to include overshooting within the framework of the local MLT since overshooting is a nonlocal phenomenon.

In this paper, we use the results of the three-dimensional numerical simulations of turbulence to calculate the convective temperature gradient and its derivatives needed in solar model calculations. As MLT is valid in most of the convective zone, it is convenient to include turbulence within the framework of MLT. In $\S 2$ we summarize the new results $\left(c_{\mu}=0.2\right)$ of the three-dimensional simulations of the solar SAL, at three stages of its evolution. These simulations are similar to those by Robinson et al. (2001) but with lower viscosities. Section 3 describes how to calculate the convective temperature gradient using turbulent velocities. We describe the calibrated solar models with turbulence in $\S 4$. In $\S 5$ the influence of turbulence on the structure variables, the adiabatic sound speed, the structure of the highly superadiabatic layer, and the $p$-mode frequencies are calculated and compared to the observed solar $p$-mode frequencies. Concluding remarks are presented in $\S 6$.

\section{TURBULENT VELOCITIES}

We incorporate the radiative hydrodynamical simulations of the outer layers of the Sun into the one-dimensional stellar models. Three three-dimensional simulations have been performed using the hydrostatic one-dimensional stellar models, at three stages of its evolution (ZAMS, present Sun, and subgiant), as starting points. The physics (thermodynamics, the equation of state, and opacities) in the simulation is the same as in the one-dimensional stellar models. These simulations follow closely the approach described by Kim \& Chan (1998) and are described in more detail by Robinson et al. (2000). The full hydrodynamical equations were solved in a thin subsection of the stellar model, i.e., a three-dimensional box located in the vicinity of the photosphere. For the radiative transport, the diffusion approximation was used in the deep region $\left(\tau>10^{3}\right)$ of the simulation, while the three-dimensional Eddington approximation was used (Unno \& Spiegel 1966) in the region above. After the simulation had reached a steady state, statistical integrations were performed for each simulation for over $2500 \mathrm{~s}$ in the case of the solar surface convection.

Turbulence can be measured by the turbulent Mach number $\mathscr{M}=v^{\prime \prime} / v_{s}$, where $v^{\prime \prime}$ is the turbulent velocity and $v_{s}$ is the sound speed. The MLT is valid when $\mathscr{M}$ is sufficiently small. In the outer layers of the Sun $\mathscr{M}$ can be of order unity (Cox \& Giuli 1968), but in the deep convection region $\mathscr{M}$ is almost zero. The turbulent velocity is defined by the velocity variance

$$
v_{i}^{\prime \prime}=\left(\overline{v_{i}^{2}}-{\overline{v_{i}}}^{2}\right)^{1 / 2},
$$

where the overbar denotes a combined horizontal and temporal average, and $v_{i}$ is the total velocity. Figure 1 shows the run of $\mathscr{M}$ as a function of $\log P$ (in base 10) in the convection simulations for the ZAMS model, the present Sun, and the subgiant model, respectively. We note that the maximum of $\mathscr{M}$ is about 0.7 and changes little from the ZAMS to the present Sun. Using $\mathscr{M}$, we can define the turbulent kinetic energy per unit mass $\chi$ as

$$
\chi=\frac{1}{2} \mathscr{M}^{2} v_{s}^{2} .
$$




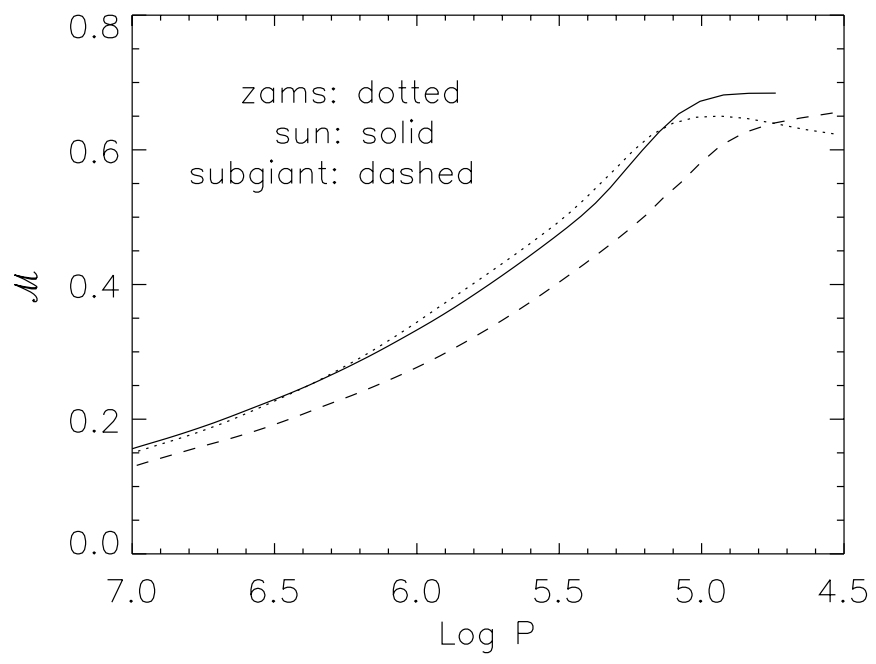

Fig. 1.-Turbulent Mach number as a function of depth at three evolutionary stages.

The turbulent contribution to the entropy is

$$
S_{\text {turb }}=\chi / T,
$$

where $T$ is the gas temperature.

Turbulence in the stratified layers of the solar convection zone is not isotropic. For convenience, we define the parameter $\gamma$ to reflect the anisotropy of turbulence,

$$
P_{\text {turb }}=(\gamma-1) \rho \chi,
$$

where $\rho \chi$ is the turbulent kinetic energy density. Since $P_{\text {turb }}=\rho v_{z}^{\prime \prime 2}$, we can relate $\gamma$ to the turbulent velocity as follows:

$$
\gamma=1+2\left(v_{z}^{\prime \prime} / v^{\prime \prime}\right)^{2}
$$

$\gamma=5 / 3$ when turbulence is isotropic $\left(v_{z}^{\prime \prime}=v_{x}^{\prime \prime}=v_{y}^{\prime \prime}\right) ; \gamma=3$ or $\gamma=1$ when turbulence is completely anisotropic $\left(v_{z}^{\prime \prime}=v^{\prime \prime}\right.$ or $v_{z}^{\prime \prime}=0$, respectively). The physical meaning of $\gamma$ is the specific heat ratio owing to turbulence. This affects the radial turbulent pressure distribution. Figure 2 shows $P_{\text {turb }}$, in which the radial turbulent pressure is scaled with the gas

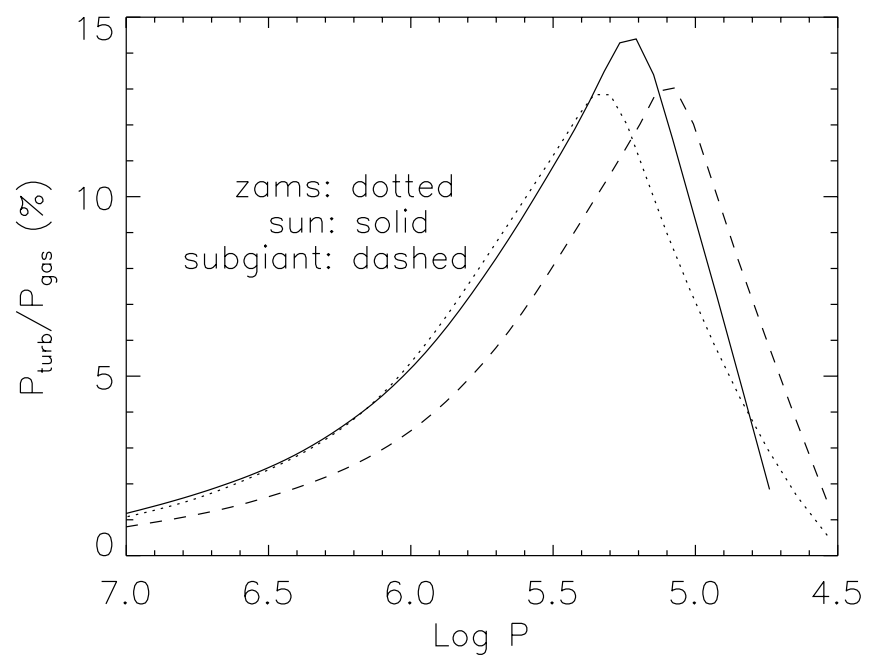

FIG. 2.-Ratio of turbulent to total pressure in the outer layers as a function of depth at three evolutionary stages.

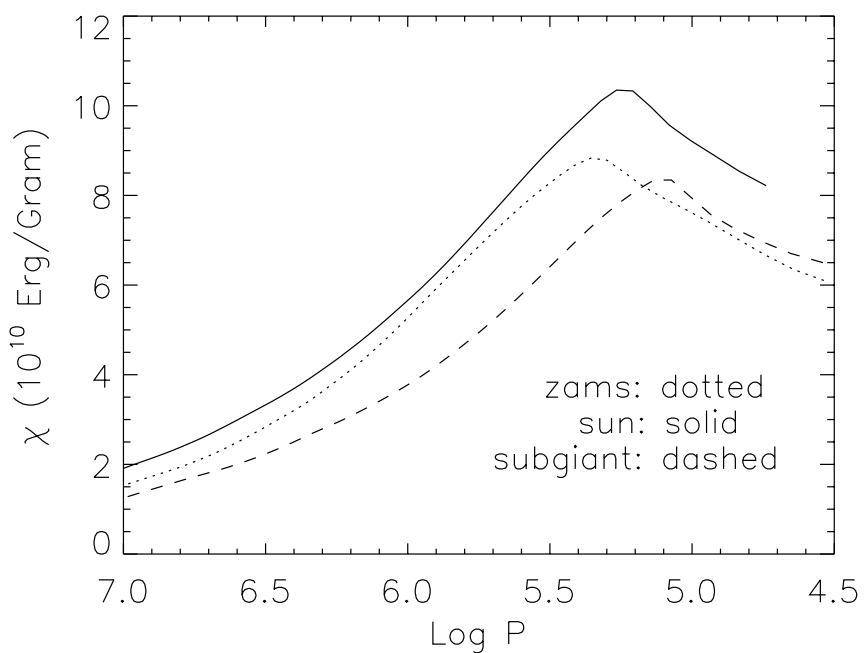

FIG. 3.-Turbulent kinetic energy per unit mass as a function of depth at three evolutionary stages.

pressure, $P_{\text {gas }}$. The total pressure is defined as

$$
P_{T}=P_{\text {gas }}+P_{\text {rad }}+P_{\text {turb }} \text {. }
$$

Note that $P_{\text {turb }} / P_{\text {gas }}=\left(v_{z}^{\prime \prime} / v_{s}\right)^{2}$ since $v_{s}=\left(P_{\text {gas }} / \rho\right)^{1 / 2}$.

The turbulent contribution to the pressure, kinetic energy, and entropy can all be expressed in terms of $\chi$ and $\gamma$. Therefore, turbulence can be parameterized by these two parameters. Figures 3 and 4 show their variations as functions of depth.

\section{CONVECTIVE TEMPERATURE GRADIENTS WITH THE TURBULENT VELOCITIES}

Abbett et al. (1997) tried to include turbulence in solar modeling within the framework of MLT by using simulated pressure and density in calculating the temperature gradient in the convection zone. As they pointed out, such an application of MLT is not self-consistent. Lydon \& Sofia (1995) developed a self-consistent method to include magnetic fields in calculating the convective temperature gradients within the framework of MLT. Then Lydon, Guenther, \& Sofia (1996) used it to successfully explain the observed

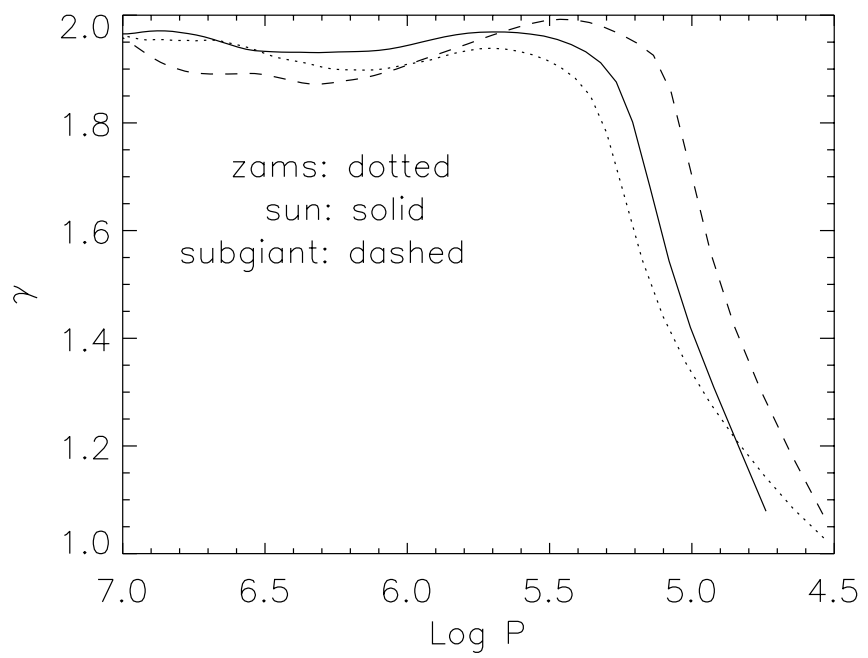

FIG. 4.- Specific heat ratio owing to turbulence as a function of depth at three evolutionary stages. 
variation of solar $p$-modes with the solar cycle. Recently, $\mathrm{Li}$ \& Sofia (2001) have updated this method to reproduce the observed cyclic variations of all solar global parameters such as solar luminosity, solar effective temperature, and solar radius. We briefly show how the same method can be used to calculate the influence of turbulence on the temperature gradients in the convection zone.

\subsection{Turbulent Variables}

Introducing $\chi$ and $\gamma$, computed from the threedimensional simulations, the equation of state becomes

$$
\rho=\rho\left(P_{T}, T, \chi, \gamma\right) .
$$

Including the turbulent kinetic energy, the first law of thermodynamics becomes

$$
\begin{aligned}
d Q_{T} & =d U+P d V+d \chi \\
& =d U_{T}+\left[P_{T}-(\gamma-1)(\chi / V)\right] d V,
\end{aligned}
$$

where $U_{T}=U+\chi$ is the total internal energy per unit mass and $V=1 / \rho$ is the volume per unit mass.

\subsection{Convective Stability Criterion}

The convective stability criterion can still be expressed by the difference between density derivatives of a mass element and its surroundings:

$$
(d \rho / d r)_{e}-(d \rho / d r)_{s}>0 .
$$

However, since we are using $P_{T}, T, \chi$, and $\gamma$ as the independent variables, we have

$$
d \rho / \rho=\mu d P_{T} / P_{T}-\mu^{\prime} d T / T-v d \chi / \chi-v^{\prime} d \gamma / \gamma,
$$

where

$$
\begin{gathered}
\mu=\left(\frac{\partial \ln \rho}{\partial \ln P_{T}}\right)_{T, \chi, \gamma}, \quad \mu^{\prime}=-\left(\frac{\partial \ln \rho}{\partial \ln T}\right)_{P_{T}, \chi, \gamma} ; \\
\nu=-\left(\frac{\partial \ln \rho}{\partial \ln \chi}\right)_{P_{T}, T, \gamma}, \quad v^{\prime}=-\left(\frac{\partial \ln \rho}{\partial \ln \gamma}\right)_{P_{T}, T, \chi} .
\end{gathered}
$$

As a result, the stability criterion becomes

$$
\nabla_{\mathrm{rad}}<\nabla_{\mathrm{ad}}-A_{m} .
$$

In this expression, $A_{m}$ reflects the direct influence of turbulence, defined by

$$
A_{m}=\left(v \nabla_{\chi}+v^{\prime} \nabla_{\gamma}\right) \nabla_{\mathrm{ad}} / \mu,
$$

where we have assumed $(d \chi / d r)_{e}=(d \chi / d r)_{s}$ and $(d \gamma / d r)_{e}=$ $(d \gamma / d r)_{s}$, while

$$
\begin{gathered}
\nabla_{\chi}=\left(\frac{\partial \ln \chi}{\partial \ln P_{T}}\right)_{s}, \quad \nabla_{\gamma}=\left(\frac{\partial \ln \gamma}{\partial \ln P_{T}}\right)_{s} ; \\
\nabla_{\mathrm{rad}}=\frac{3}{16 \pi a c G} \frac{\kappa L_{r} P_{T}}{M_{r} T^{4}}, \quad \nabla_{\mathrm{ad}}=\frac{P_{T} \delta}{\rho T c_{p}} .
\end{gathered}
$$

The other symbols have the usual meanings.

\subsection{Convective Temperature Gradients}

3.3.1. Flux Conservation with Turbulence

The convective temperature gradient $\nabla_{\text {conv }}$ is determined by the requirement that the total energy flux $F_{\text {total }}$ equals the sum of the radiative flux $F_{\text {rad }}$ and the convective flux
$F_{\text {conv }}:$

$$
F_{\text {total }}=F_{\text {rad }}+F_{\text {conv }} .
$$

The total flux at any given layer in the star is determined by the photon luminosity $L_{r}$ :

$$
F_{\text {total }}=\frac{L_{r}}{4 \pi r^{2}}=\frac{4 a c G}{3} \frac{T^{4} M_{r}}{\kappa P_{T} r^{2}} \nabla_{\text {rad }} .
$$

The radiative flux is determined by the convective temperature gradient:

$$
F_{\text {rad }}=\frac{4 a c G}{3} \frac{T^{4} M_{r}}{\kappa P_{T} r^{2}} \nabla_{\text {conv }} .
$$

The convective flux is determined by the convective velocity $v_{\text {conv }}$ and the heat excess $D Q_{T}$ :

$$
F_{\text {conv }}=\rho v_{\text {conv }} D Q_{T} .
$$

When the convective velocity is much smaller than the sound speed of the medium, the process can be considered to be of constant pressure. In this case, the heat excess can be obtained from the first law given by equation (8):

$$
D Q_{T}=c_{p} D T+\left(\frac{P_{T} \mu^{\prime} v}{\rho \mu \chi}+1\right) D \chi+\frac{P_{T} \mu^{\prime} v^{\prime}}{\rho \mu \gamma} D \gamma .
$$

\subsubsection{Mixing-Length Approximation}

Using the mixing-length approximation, $D T, D \chi$, and $D \gamma$ can be expressed by the mixing length $l_{m}$ as follows:

$$
\begin{aligned}
D T / T & =(1 / T) \partial(D T) / \partial r\left(l_{m} / 2\right) \\
& =\left(\nabla_{\text {conv }}-\nabla_{e}\right)\left(l_{m} / 2\right)\left(1 / H_{p}\right), \\
D \chi / \chi & =(1 / \chi) \partial(D \chi) \partial r\left(l_{m} / 2\right)=0, \\
D \gamma / \gamma & =(1 / \gamma) \partial(D \gamma) \partial r\left(l_{m} / 2\right)=0,
\end{aligned}
$$

where $H_{p}=-P_{T}\left(d r / d P_{T}\right)$ is the pressure scale height. In order to determine $v_{\text {conv }}$, the MLT assumes that half of the work done by half of the radial buoyancy force acted over half the mixing length goes into the kinetic energy of the element $\left(v_{\text {conv }}^{2} / 2\right)$. Since the radial buoyancy force per unit mass is related to the density difference by

$$
k_{r}=-g(D \rho / \rho),
$$

and since the process is in pressure equilibrium, we obtain

$$
v_{\mathrm{conv}}^{2}=\mu^{\prime}\left(\nabla_{\mathrm{conv}}-\nabla_{e}\right) \frac{l_{m}^{2} g}{8 H_{p}},
$$

where $g=G M_{r} / r^{2}$ is the gravitational acceleration.

An additional relation is required to close the MLT:

$$
\left(d Q_{T} / d r\right)_{e}=(\text { radiative losses })+(\text { change of } \chi),
$$

which can be expressed as

$$
\begin{aligned}
\left(2 a c T^{3}\right) /\left(\rho c_{p} v_{\text {conv }}\right) & \left(\omega /\left(1+\frac{1}{3} \omega^{2}\right)\left(\nabla_{\text {conv }}-\nabla_{e}\right)\right. \\
= & \left(\nabla_{e}-\nabla_{\mathrm{ad}}\right)+\left(\nabla_{\mathrm{ad}} / \mu\right)\left(v \nabla_{\chi}+v^{\prime} \nabla_{\gamma}\right),
\end{aligned}
$$

where $\omega=\kappa \rho l_{m}$.

$$
\text { 3.3.3. Result }
$$

Solving equations (13) and (22), we obtain

$$
\nabla_{\text {conv }}=\nabla_{\mathrm{ad}}+\left(y / V \gamma_{0}^{2} C\right)(1+y / V)-A_{m},
$$


where $y$ is the solution of the following equation:

$$
2 A y^{3}+V y^{2}+V^{2} y-V=0 .
$$

The values $A, \gamma_{0}, C$, and $V$ are defined by

$$
\begin{aligned}
A & =(9 / 8)\left[\omega^{2} /\left(3+\omega^{2}\right)\right], \\
\gamma_{0} & =\left[\left(c_{\mathrm{p}} \rho\right) /\left(2 a c T^{3}\right)\right]\left[\left(1+\frac{1}{3} \omega^{2}\right) / \omega\right], \\
C & =\left(g / l_{m}^{2} \mu^{\prime}\right) / 8 H_{p}, \\
V & =1 /\left[\gamma_{0} C^{1 / 2}\left(\nabla_{\mathrm{rad}}-\nabla_{\mathrm{ad}}+A_{m}\right)^{1 / 2}\right] .
\end{aligned}
$$

From these formulae it can be seen that the effect of turbulence on the temperature gradient in the convection zone can be taken into account by modifying the adiabatic gradient:

$$
\nabla_{\mathrm{ad}}^{\prime}=\left[1-\left(\nu \nabla_{\chi}+v^{\prime} \nabla_{\gamma}\right) / \mu\right] \nabla_{\mathrm{ad}} .
$$

Therefore, it is easy to include this effect in the standard stellar structure codes.

\section{CALIBRATED SOLAR MODELS}

\subsection{Standard Solar Model}

For the purpose of comparison, we construct an SSM with the Yale stellar evolution code. The OPAL opacities tables (Iglesias \& Rogers 1996) are used together with the low-temperature opacities from Alexander \& Ferguson (1994). The equation of state is taken from Rogers, Swenson, \& Iglesias (1996). When out of the table, the Yale standard implementation with the Debye-Hükel correction is used (Guenther et al. 1992). Helium and heavy-element diffusion processes are included in the model. Heavyelement diffusion is implemented by assuming that all heavy elements diffuse with the same velocity as fully ionized iron (Guenther \& Demarque 1997). The model atmosphere is constructed using the empirical Krishna-Swamy $T$ - $\tau$ relation (Guenther et al. 1992). The solar model is evolved from the ZAMS to the current solar age. The mixing-length ratio $\alpha$ and the helium content $Y$ have been adjusted in the usual way so as to match the solar luminosity, radius, and the ratio of heavy elements to hydrogen $(Z / X=0.0230)$ at the surface of the model (Grevesse \& Sauval 1998). These are obtained by choosing $\left(Z_{0}, X_{0}\right)$ and $\alpha$ to be $(0.0188$, 0.7091724 ) and 2.135772 . The standard (or reference) solar model is abbreviated as the SSM.

\subsection{Solar Model with Turbulent Pressure Alone}

The simplest way to taken into account turbulence in solar modeling is to include turbulent pressure (or Reynolds stress) alone, as done by many authors (e.g., Balmforth 1992a). In this case, only the hydrostatic equilibrium equation needs to be modified as follows (method 1$)$ :

$$
\frac{\partial P}{\partial M_{r}}=-\frac{G M_{r}}{4 \pi r^{4}}(1+\beta),
$$

where $P=P_{\text {gas }}+P_{\text {rad }}$, and

$$
\beta=\left(\frac{2 P_{\text {turb }}}{\rho g r}-\frac{\partial P_{\text {turb }}}{\partial P}\right)\left(1+\frac{\partial P_{\text {turb }}}{\partial P}\right)^{-1} .
$$

Here $2 P_{\text {turb }} /(\rho g r)$ originates from the spherical coordinate system adopted, representing a kind of geometric effect. The equations that govern the envelope integrations also need to be changed accordingly.
We implement this case by modifying the Yale stellar evolution code and obtain a nonstandard model in the same way we obtain the standard model. We assume $P_{\text {turb }}$, set equal to its value for the present Sun, does not change from the ZAMS to the present age of the Sun. The adjustable parameters now are fixed as $\left(Z_{0}, X_{0}, \alpha\right)=(0.0188$, $0.7092889,2.138190)$. This model is called the pressure solar model (PSM).

\subsection{Solar Model with $\chi$ and $\gamma$ as Independent Variables}

The form of the continuity equation and the equation of transport of energy by radiation is not affected by turbulence. The hydrostatic equation includes a Reynolds stress term owing to turbulence:

$$
\frac{\partial P}{\partial r}=-\frac{G M_{r}}{r^{2}} \rho-\frac{1}{r^{2}} \frac{d}{d r}\left(r^{2} \rho v_{r} v_{r}\right),
$$

where $P=P_{\text {gas }}+P_{\text {rad }}$. Since the last term can be rewritten as $\partial P_{\text {turb }} / \partial r+2(\gamma-1) \chi / r$, this equation becomes

$$
\frac{\partial P_{T}}{\partial M_{r}}=-\frac{G M_{r}}{4 \pi r^{4}}-\frac{2(\gamma-1) \chi}{4 \pi r^{3}} .
$$

The last term on the right-hand side of equation (29) also embodies the same spheric geometric effect as $2 P_{\text {turb }} /(\rho g r)$ in equation (27). The energy conservation equation is also affected by turbulence because the first law of thermodynamics should include the turbulent kinetic energy

$$
\frac{\partial L_{r}}{\partial M_{r}}=\epsilon-T \frac{d S_{T}}{d t},
$$

where

$$
T d S_{T}=c_{p} d T-\frac{\mu^{\prime}}{\rho} d P_{T}+\left(1+\frac{P_{T} \mu^{\prime} v}{\rho \mu \chi}\right) d \chi+\frac{P_{T} \mu^{\prime} v^{\prime}}{\rho \mu \gamma} d \gamma .
$$

The equation of energy transport by convection,

$$
\frac{\partial T}{\partial M_{r}}=-\frac{T}{P_{T}} \frac{G M_{r}}{4 \pi r^{4}} \nabla_{\text {conv }},
$$

does not change in form, but the convection temperature gradient, obtained in the previous section, is different from that without turbulence. The equations that govern envelope integrations also need to be changed accordingly. This method will be referred to below as method 2 .

We implement this case by modifying the Yale stellar evolution code. Once again, we obtain a nonstandard model in the same way as we obtained the standard model. If we assume that $\chi$ and $\gamma$ do not change with time (letting them equal their values at the present age of the Sun), the adjustable parameters now must be set as $\left(Z_{0}, X_{0}, \alpha\right)=$ $(0.0188,0.7092715,2.271540)$. We use the spline interpolation of $\chi$ and $\gamma$ given in $\S 2$ for their pressure dependence in the model calculations. We call this model the Energy Solar Model 1 (ESM1).

In order to investigate the evolutionary effects of $\chi$ and $\gamma$, we linearly interpolate between the two simulations that are closest to the evolutionary state of the model. In this case, $\left(Z_{0}, X_{0}, \alpha\right)=(0.0188,0.7092945,2.271462)$ in order to match the observed solar luminosity, radius, and ratio of 
heavy elements to hydrogen $(Z / X)$. We call this model the Energy Solar Model 2 (ESM2).

\section{INFLUENCE OF TURBULENCE ON THE SOLAR STRUCTURE}

We shall now investigate how different methods for including turbulence in solar modeling affect the solar model structure.

\subsection{Measured by Structural Variables}

Figure 5 depicts turbulence-induced relative changes (with respect to the SSM) of the solar structural variables for the PSM (dotted line), ESM1 (dashed line), and ESM2 (solid line), as functions of the base-10 logarithm of the total pressure. The changes for the pressure, temperature, and luminosity are calculated at the same radius coordinate, while the radius change is calculated at the same interior mass coordinate $M_{r}$. From this figure the following can be seen:

1. In all the cases turbulence affects the distribution of the pressure, temperature, and the (radiative and convective, rather than the total) luminosity around the SAL peak (specified by the dashed vertical line in the figure).

2. Both methods produce almost the same maximum pressure change of $15 \%$, but method 1 produces a larger change for the temperature ( $8 \%$ vs. $4 \%)$, radiative and convective luminosity ( $130 \%$ vs. $70 \%$ and $100 \%$ vs. $80 \%)$.

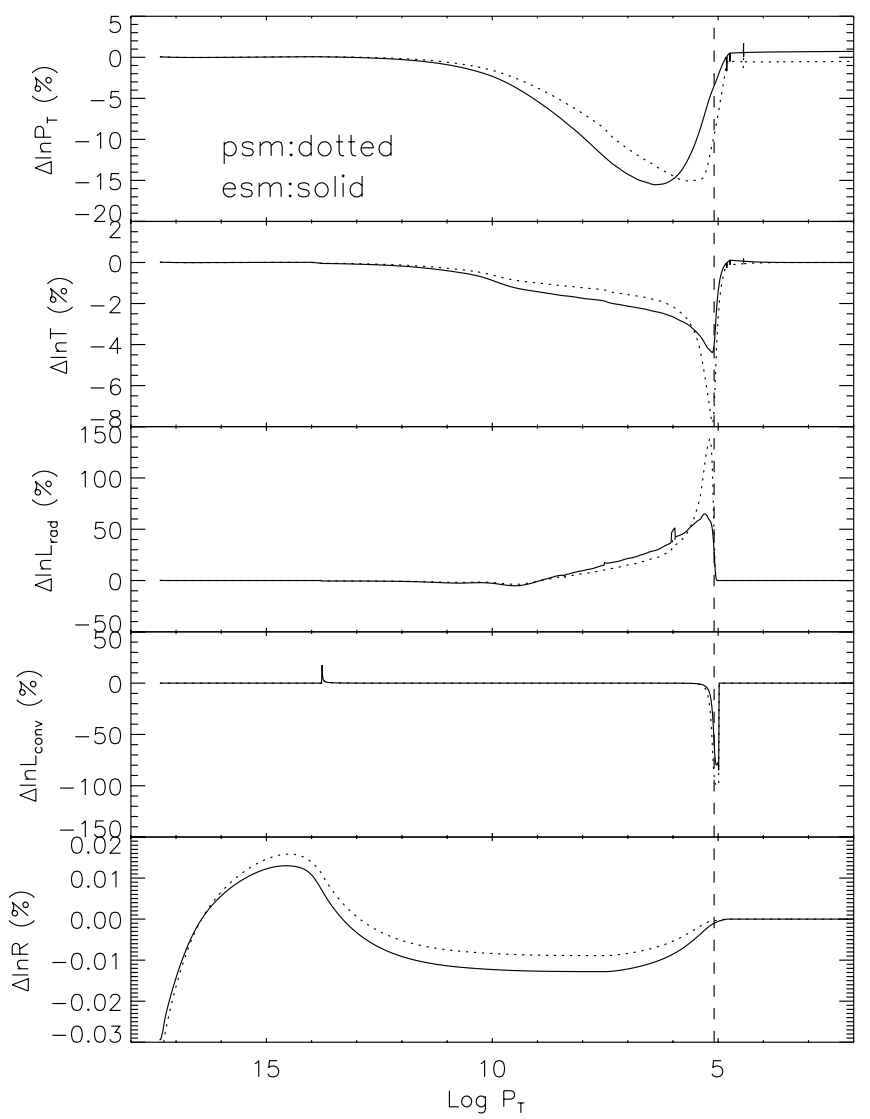

Fig. 5.-Turbulence-induced relative changes of the solar structural variables (pressure, temperature, radiative luminosity, convective luminosity, and radius) for the PSM and ESM with respect to the SSM. The vertical line indicates the location of the SAL peak of the SSM: $\log P_{T}=$ 5.09 .
3. It is surprising that turbulence near the surface affects (slightly) the mass distribution (denoted by the radius change) in the core of the solar models.

The increase of the radiative luminosity does not necessarily imply the increase of the convective gradient since the radiative flux depends on not only the convective gradient, but also the temperature $T$, the radiative opacity $\kappa$, and the pressure $P_{T}$, as expressed by equation (15). Similarly, the decrease of the convective luminosity does not necessarily imply the increase of the convective gradient since the convective flux depends on the specific heat at constant pressure $c_{p}$, density $\rho$, temperature $T$, convective velocity $v_{\text {conv }}$, and the mixing length $l_{m}$ :

$$
F_{\text {conv }}=\frac{4 c_{p} \rho T}{g \mu^{\prime} l_{m}} v_{\text {conv }}^{3} .
$$

Figure 6 shows how $\rho, \kappa, v_{\text {conv }}, l_{m}$, and $c_{p}$ in the PSM and ESM change with respect to the SSM. The increase of the radiative luminosity below the SAL peak is mostly generated by the decrease of the radiative opacity caused by the decrease of the temperature and density. The decrease of the convective luminosity above the SAL peak is mostly generated by the decrease of the convective velocity.

Figures 5 and 6 show that the temperature, density, and total pressure decrease when the turbulent pressure is included. The decrease of the total pressure is possible since what supports gravity in the solar interior is the total pres-

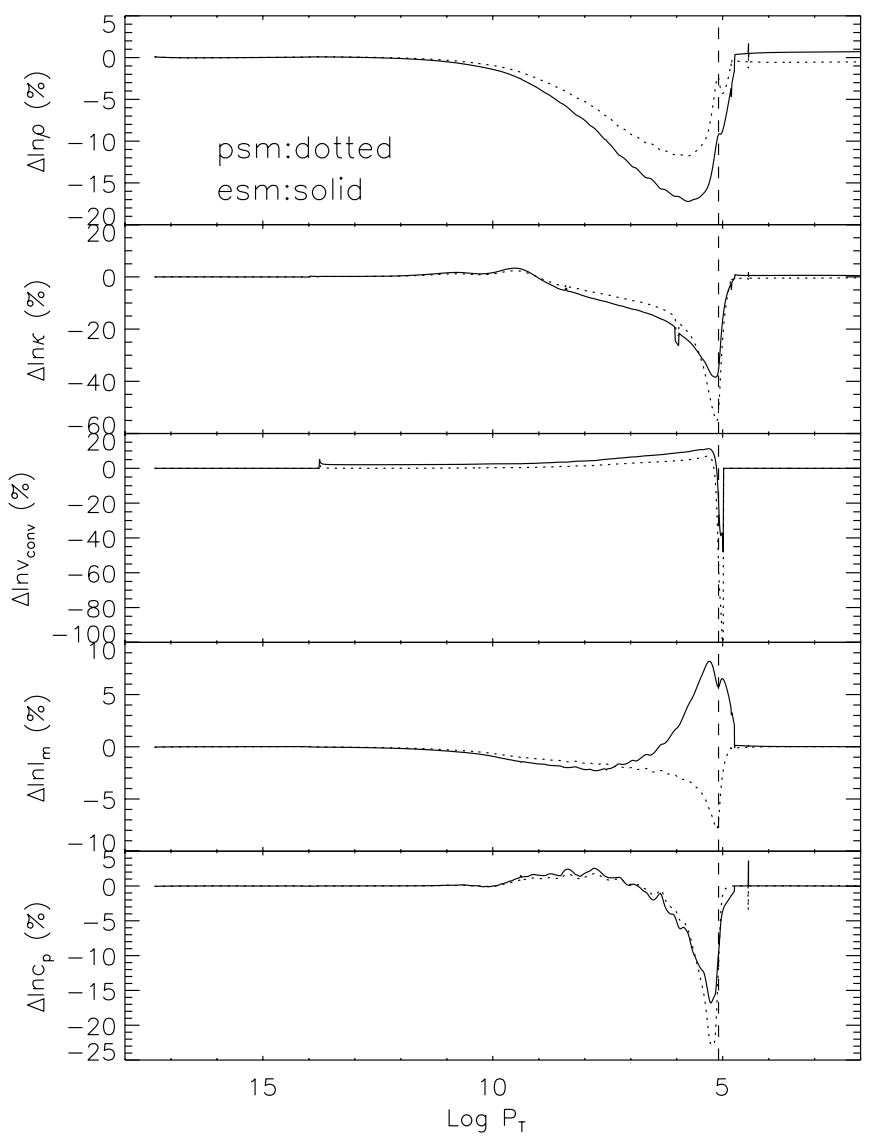

FIG. 6.- Relative change of the density, opacity, convective velocity, mixing length, and specific heat at the constant pressure in the PSM (dotted line) and ESM (solid line) with respect to the SSM. 
sure gradient, not the pressure itself. Besides, the gas pressure may decrease in order to maintain hydrodynamic equilibrium when turbulence provides a turbulent pressure. Consequently, the gas density and/or the gas temperature should decrease. However, the two methods make a difference here: the decrease of the temperature (density) in the ESM is smaller (larger) than that in the PSM. The cause is that the mixing length in the ESM increases near the SAL peak, but the mixing length in the PSM decreases near the SAL peak. This implies that the transport of energy by convection near the peak in the ESM is more efficient than that in the PSM. As a result, different SAL structures are expected, as addressed in the next subsection.

The comprehensive effect of turbulence on solar structure manifests itself in the adiabatic sound speed

$$
\Delta \ln C=\frac{1}{2}\left(\Delta \ln \Gamma_{1}+\Delta \ln P_{T}-\Delta \ln \rho\right),
$$

where $\Gamma_{1}$ is the first adiabatic exponent defined by

$$
\Gamma_{1}=\left(\frac{\partial \ln P_{T}}{\partial \ln \rho}\right)_{s, \chi, \gamma}
$$

when turbulence is modeled by $\chi$ and $\gamma$. Figure 7 shows these four quantities for the ESM and PSM. Obviously, the pressure and density changes contribute to the change of the adiabatic sound speed, as does the change of the first adiabatic exponent. This shows that the thermodynamic properties of the solar matter changes when turbulence is present, as expected. Nevertheless, the two methods produce a difference for the first adiabatic exponent around the SAL peak.

Another feature that we can see from Figures 5-7 [the relative change for any variable $X$ is defined as follows: $\left.\left(X_{\text {model }}-X_{\mathrm{ssm}}\right) / X_{\mathrm{ssm}}\right]$ is that although turbulence is restricted to the highly superadiabatic layer $\left(\log P_{T} \in\right.$ (4.6, 7); see Figs. 1-4), its influence extends deeply into

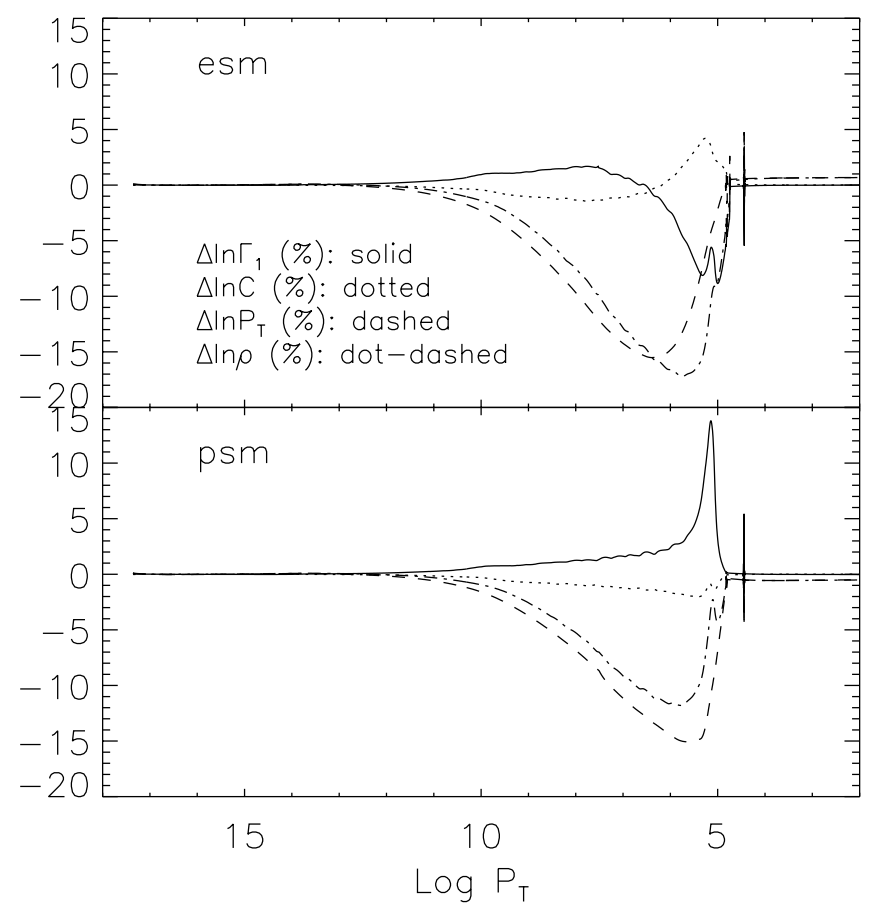

FIG. 7.- Relative change of the adiabatic sound speed and its contributors in the ESM and PSM. the solar interior for the ESMs. For example, we still see some influence near the base of the convective zone at $\log P_{T} \sim 13$. This is a natural consequence of continuity.

\subsection{Measured by Superadiabaticity}

The superadiabaticity $\left(\nabla-\nabla_{\mathrm{ad}}\right)$ as a function of the base-10 logarithm of total pressure is depicted in Figure 8 for the SSM (solid line) and ESM1 (dotted line). The PSM has the same SAL as the SSM, and ESM2 has the same SAL as the ESM1. The SAL peak of the SSM equals 0.45, while that of the ESM equals 0.40 , about $11 \%$ lower than that of the SSM.

The corresponding three-dimensional simulations produce an SAL very similar to the one-dimensional model. The maximum of $\nabla-\nabla_{\mathrm{ad}}$ is about 0.4. The threedimensional solar surface simulations by Stein $\&$ Nordlund (1998; Rosenthal et al. 1999) and Canuto's one-dimensional turbulence models found a much higher peak of about 0.8 . The simulations by Stein and Nordlund employ a hyperviscosity model for the subgrid scales, while our threedimensional simulations use the Smagorinsky model (Smagorinsky 1963) with the smallest viscosity that was numerically stable $\left(c_{\mu}=0.2\right.$; see below). If we increased the viscosity by an order of magnitude, then the SAL peak was similar to Stein and Nordlund's.

In order to understand these results, we note that the actual temperature gradient is determined by the relative efficiency between the radiative and convective transport of heat since the total energy flux is fixed by the total luminosity (see eq. [14]). Figure 9 shows the actual and adiabatic temperature gradients for the ESM and PSM, from which we can see that the SAL peak decrease in the ESM with respect to the SSM is mostly caused by the decrease of the actual gradient owing to the inclusion of the turbulent kinetic energy. The maximum relative change of the convective (and adiabatic) gradient in the ESM is less than $10 \%$. In the MLT approach, the convective flux is proportional to the convective velocity, as shown by equation (16). In threedimensional simulations, the total flux is equal to the radiative flux $F_{\text {rad }}$, plus the enthalpy flux $F_{e}$, plus the turbulent kinetic energy flux $F_{k}$. Near the SAL peak, $F_{k} \approx 0$. The enthalpy flux (Chan \& Sofia 1989) is proportional to the rms fluctuation of vertical velocity $v_{z}^{\prime \prime}$ defined in

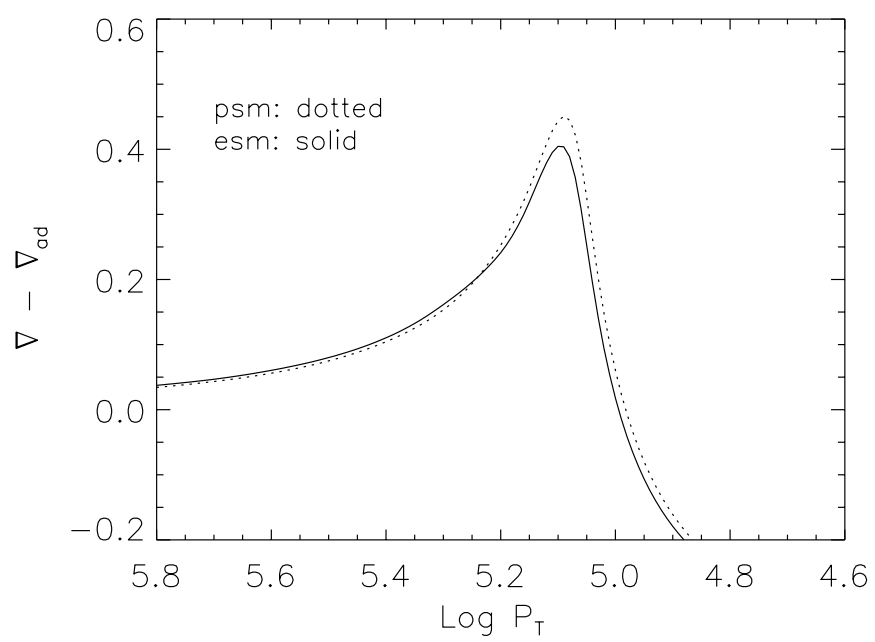

FIG. 8.-Structure of the highly superadiabatic layer. The SAL of the SSM overlaps that of the PSM. 


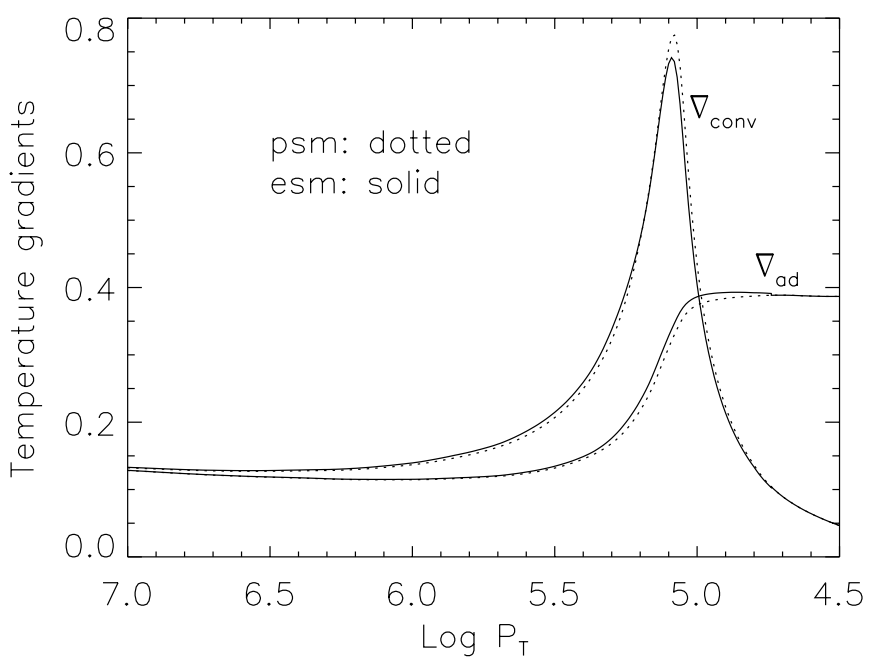

FIG. 9.-Convective and adiabatic temperature gradients. Those of the SSM and PSM overlap each other.

equation (1),

$$
F_{e}=\rho c_{p} \overline{v_{z}^{\prime} T^{\prime}}=\rho c_{p} C\left(v_{z}^{\prime} T^{\prime}\right) v_{z}^{\prime \prime} T^{\prime \prime},
$$

where $C\left(v_{z}^{\prime} T^{\prime}\right)$ is the correlation coefficient between the temperature and vertical velocity fluctuations, and $T^{\prime \prime}$ is the rms fluctuation of temperature. Since $F_{k} \approx 0$ near the SAL peak, the decrease of the convective gradient in the ESM and the three-dimensional simulations implies an increase of the convective velocity $v_{\text {conv }}$ and the rms fluctuation of the vertical velocity just near the peak, as confirmed by Figure 10. This shows that the SAL peak is sensitive to the turbulent velocity.

The most reliable method to determine the turbulent velocity is by direct numerical simulations (DNS) using the real solar kinematic viscosity $v$ (Landau \& Lifshitz 1987). Since the number of degrees of freedom needed to represent three-dimensional turbulent convection is proportional to $\mathrm{Re}^{9 / 4}$, to resolve numerically all the scales in the solar convection zone (where the Reynolds number $\mathrm{Re} \approx 10^{12}$ ) would require $10^{27}$ grid points (Canuto 2000). However, the

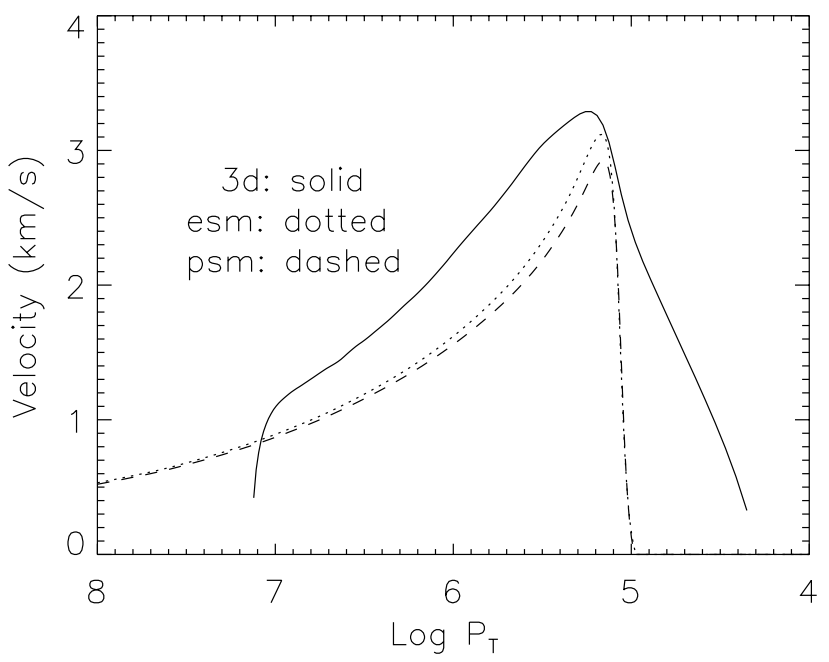

Fig. 10.-Radial turbulent velocity and convective velocity as a function of depth (measured by the base-10 logarithm of pressure). Those of the SSM and PSM overlap each other. maximum number of grid points allowed by the present technology in DNS is $O\left(10^{9}\right)$. This forces us to use large eddy simulations (LES) by increasing the kinematic viscosity $v$ so that it represents the effects of Reynolds stresses on the unresolved or subgrid scales (SGS). In the LES simulations, we used the SGS formula owing to Smagorinsky (1963),

$$
v=\left(c_{\mu} \Delta\right)^{2}(2 \sigma: \sigma)^{1 / 2} .
$$

The colon inside the brackets denotes tensor contraction of the rate of strain $\sigma_{i j}=\left(\nabla_{i} \bar{v}_{j}+\nabla_{j} \bar{v}_{i}\right) / 2, \Delta$ is the grid spacing, and $c_{\mu}$ is an adjustable dimensionless parameter. In the Smagorinsky model, $v \approx\left(c_{\mu} \Delta\right)^{2} u / L$, where the characteristic speed $u$ and length $L$ are estimated from the resolved motions. Consequently, the Reynolds number reads

$$
\operatorname{Re}=\frac{u L}{v}=\left(\frac{L}{c_{\mu} \Delta}\right)^{2}
$$

In nondimensional units, $L \approx 1, c_{\mu}=0.2$, and $\Delta \approx 0.01$. So $\operatorname{Re} \sim 10^{6}$. This number is still much smaller than the Reynolds number $10^{12}$ in the solar convection zone. Therefore, the viscosity must be overestimated, although this Reynolds number should be high enough for the fluid to be turbulent. The fact that the standard MLT generates a lower convective velocity may imply that the MLT assumes a larger viscosity than the three-dimensional simulations. This consideration disfavors those solar models with a higher SAL peak than that of the SSM.

It should be pointed out that we compare different solar models at the same radius coordinate, while we compare the SAL at the same pressure coordinate. Therefore, we observe a large difference for the convective velocity in Figure 6 but a small (or no) difference between the ESM (PSM) and the SSM in Figure 9.

\subsection{Measured by $p$-Mode Oscillation Frequencies}

Our principal goal is to investigate how the treatment of turbulence in solar modeling affects the computed model structure. Therefore, we do not include the contribution of turbulence to the pulsation equations when we calculate $p$-mode oscillation frequency differences caused by turbulence. We use Guenther's (1994) pulsation code to calculate the $p$-mode frequencies under the adiabatic approximation, for our SSM, PSM, ESM1, and ESM2, respectively.

Figure 11 shows the frequency differences (turbulent solar model, including the PSM, ESM1, and ESM2, minus SSM) scaled by the mode mass $Q_{\mathrm{n} 1}$ (e.g., Christensen-Dalsgaard \& Berthomieu 1991). The frequency differences between the PSM and SSM are comparable with Balmforth's (1992a, Table 1). Both models use turbulent pressure alone. The frequency differences between the ESM and SSM are comparable with Rosenthal et al.'s (1999, Fig. 5).

In order to examine if the frequency differences shown in Figure 11 are caused by the calibration of the solar models, we calculated the corresponding uncalibrated models for the calibrated PSM and ESM2, respectively. Unlike the calibrated model, the starting model for the uncalibrated model is the SSM at the present age of the Sun, instead of the ZAMS model. We switch on the turbulence and evolve the model 10 time steps with $1 \mathrm{yr}$ as the time step length. The frequency differences between calibrated and uncalibrated turbulent models are less than $1 \mu \mathrm{Hz}$.

By calculating the frequency differences between the ESM1 and ESM2 models we can find out the effect of tem- 


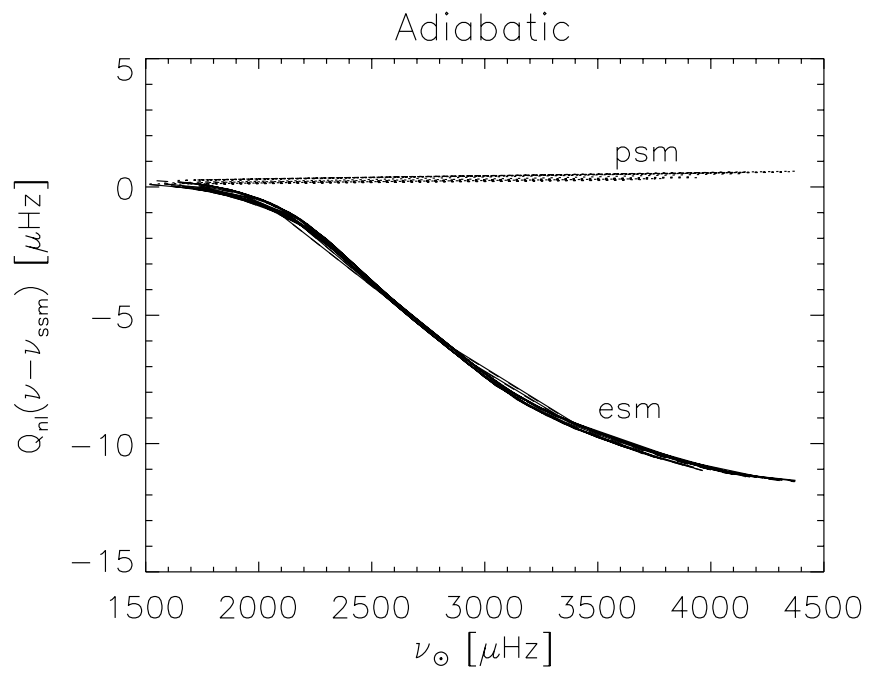

FIG. 11. $-p$-mode frequency difference diagrams: Turbulent model minus standard model, for the turbulent PSM, and the solar model with the turbulent pressure and turbulent kinetic energy (ESM1 and ESM2). Plotted are the $l=0,1,2,3,4,10,20, \ldots, 100 p$-modes.

poral change of turbulence in the evolutionary timescale of the Sun. From Figure 11 we can see that this effect is very small $(<0.5 \mu \mathrm{Hz})$.

As pointed out earlier, the PSM model is obtained by including turbulent pressure alone in the solar modeling, while the ESMs are obtained by introducing the turbulent variables $\chi$ and $\gamma$ to include both turbulent pressure and kinetic energy. Therefore, the frequency differences between these two kinds of models reflect the different treatments of turbulence in the solar modeling. Physically, the differences are caused by the addition of turbulent kinetic energy. Figure 11 shows that the frequency differences caused by turbulent kinetic energy are much larger than those caused by turbulent pressure alone in size. Figure 12 shows that the frequency changes caused by turbulent kinetic energy make

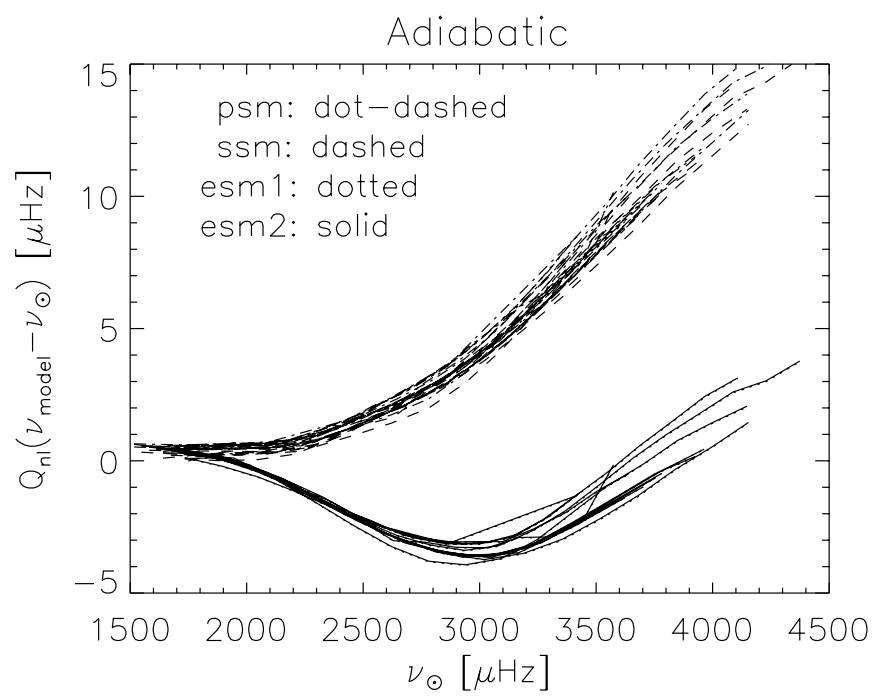

FIG. 12. - $p$-mode frequency difference diagrams: observation minus model, scaled by the mode mass $Q_{\mathrm{n}}$, for the SSM, the turbulent PSM, the solar model with fixed turbulent pressure and kinetic energy (ESM1), and the solar model with evolutionary turbulent pressure and kinetic energy (ESM2, almost overlapping ESM1). Plotted are the $l=0,1,2,3,4,10,20$, ..., $100 p$-modes. the computed model frequencies match the solar data better than the SSM model, which is in agreement with Rosenthal et al.'s result (1999, Figs. 1 and 6).

\section{CONCLUDING REMARKS}

We have shown how different treatments of turbulence in solar modeling affect the model structure within the framework of the standard MLT. The turbulent velocity is obtained from three-dimensional numerical simulations of turbulence in the highly superadiabatic layer of the Sun. When we introduce the turbulent kinetic energy per unit mass $\chi$ and the effective ratio of specific heats owing to the turbulent perturbation $\gamma$ as independent thermodynamic variables, the resultant solar model is in agreement with the patched solar model, in which the simulated SAL replaces the original SAL. The frequency shift tends to match the observations better than the SSM. In contrast, when we use only the turbulent pressure, the turbulent effects are substantially underestimated (in the sense that the resultant $p$-mode frequency shift is much smaller).

Another difference between methods 1 and 2 is that the SAL peak in the ESM is lower than that of the SSM, but that of the PSM is the same as that of the SSM. The reason is that the increase of the mixing length, in the vicinity of the SAL peak, by the turbulent kinetic energy, is more than double the reduction of the mixing length by the turbulent pressure (see panel 4 of Fig. 6). The SAL produced by method 2 is consistent with that of our three-dimensional simulations, while method 1 does not change the SAL structure of the SSM.

Previously, the turbulent pressure was considered to play an important role in solar modeling, but we have shown that this is not true: it is the turbulent kinetic energy that is important. In fact, if we calibrate the solar model, the elevation caused by the turbulent pressure $\Delta r$ vashishes. Consequently,

$$
\frac{\delta v}{v} \approx \frac{\Delta r / c_{\mathrm{ph}}}{\int_{0}^{R \odot} d r / C}
$$

vanishes, where $c_{\mathrm{ph}}$ is the adiabatic sound speed at the solar surface (photosphere). However, we obtain almost the same frequency changes with and without calibrating the model radius to the solar radius at the present age of the Sun. Moreover, the solar model with the turbulent pressure alone does not reproduce the $p$-mode frequency shift obtained by Rosenthal et al.'s patched model (1999).

To match the observed solar $p$-mode frequencies is not the only motivation to improve the solar model by including turbulence. More interesting is to generate a solar model that can excite the observed $p$-modes and damp the unobserved modes simultaneously. Such an effort is in progress (L. H. Li et al. 2002, in preparation). Overshooting may also play an important role in this regard.

This research was supported in part by a grant from NASA and in part by Natural Science Foundation of China (project 19675064, L. H. Li). Support from NASA grant NAG 5-8406 to Yale University, and from the National Science and Engineering Research Council of Canada (D. B. Guenther) are also gratefully acknowledged. Helpful comments from S. Basu are gratefully acknowledged. 
REFERENCES

Abbett, W. P., Beaver, M., Davis, B., Georgobiani, D., Rathbun, B., \& Stein, R. F. 1997, ApJ, 480, 395

Alexander, D. R., \& Ferguson, J. W. 1994, ApJ, 437, 879

Balmforth, N. 1992a, MNRAS, 255, 603

$$
\text { . 1992b, MNRAS, 255, } 632
$$

1992c, MNRAS, 255, 639

Canuto, V. M. 1990, A\&A, 227, 282

$$
\text { 1996, ApJ, 467, } 385
$$

- 2000, ApJ, 541, L79

Canuto, V. M., Goldman, I., \& Mazzitelli, I. 1996, ApJ, 473, 550

Canuto, V. M., \& Mazzitelli, I. 1991, ApJ, 370, 295

$$
\text { 1992, ApJ, 389, } 724
$$

Chan, K. L., \& Sofia, S. 1989, ApJ, 336, 1022

Christensen-Dalsgaard, J., \& Berthomieu, G. 1991, in Solar Interior and Atmosphere, ed. A. N. Cox, W. C. Livingston, \& M. Mathews (Tucson: Univ. Arizona Press), 401

Cox, J. P., \& Giuli, R. T. 1968, Principles of Stellar Structure (New York: Gordon \& Breach)

Demarque, P., Guenther, D. B., \& Kim, Y.-C. 1997, ApJ, 474, 790 1999, ApJ, 517, 510

Grevesse, N., \& Sauval, A. J. 1998, Space Sci. Rev., 85, 161

Guenther, D. B. 1994, ApJ, 422, 400

Guenther, D. B., \& Demarque, P. 1997, ApJ, 484, 937

Guenther, D. B., Demarque, P., Kim, Y.-C., \& Pinsonneault, M. H. 1992, ApJ, 387, 372

Iglesias, C. A., \& Rogers, F. J. 1996, ApJ, 464, 943
Kim, Y.-C. 1993, Ph.D. thesis, Yale Univ.

Kim, Y.-C., \& Chan, K. L. 1997, in SCORe '96: Convection and Oscillations and Their Relationship, ed. F. P. Pijpers, J. ChristensenDalsgaard, \& C. S. Rosenthal (Dordrecht: Kluwer), 131

. 1998, ApJ, 496, L121

Kim, Y.-C., Fox, P. A., Demarque, P., \& Sofia, S. 1996, ApJ, 461, 499

Kim, Y.-C., Fox, P. A., Sofia, S., \& Demarque, P. 1995, ApJ, 442, 422

Landau, L. D., \& Lifshitz, E. M. 1987, Fluid Mechanics (Oxford: Pergamon Press), 47

Li, L. H., \& Sofia, S. 2001, ApJ, 549, 1204

Lydon, T. J. 1993, Ph.D. thesis, Yale Univ.

Lydon, T. J., Fox, P. A., \& Sofia, S. 1992, ÀpJ, 397, 701

Lydon, T. J., Guenther, D. B., \& Sofia, S. 1996, ApJ, 456, L127

Lydon, T. J., \& Sofia, S. 1995, ApJS, 101, 357

Niemela, J. J., Skrbek, L. Sreenivasan, K. R., \& Donnelly, R. J. 2000, Nature, 404, 837

Robinson, F. J., Demarque, P., Sofia, S., Chan, K. L., Kim, Y.-C., \& Guenther, D. B. 2001, in Proc. SOHO 10/GONG 2000 Workshop, Helio- and Astroseismology at the Dawn of the Millenium, ed. A. Wilson (ESA SP-464; Noordwijk: ESA), 443

Rogers, F. J., Swenson, F. J., \& Iglesias, C. A. 1996, ApJ, 456, 902

Rosenthal, C. S., Christensen-Dalsgaard, J., Nordlund, Å., Stein, R. F., \& Trampedach, R. 1999, A\&A, 351, 689

Smagorinsky, J. 1963, Mon. Weather Rev., 91, 99

Stein, R. F., \& Nordlund, A. 1998, ApJ, 499, 914

Unno, W., \& Spiegel, E. A. 1966, PASJ, 18, 85 